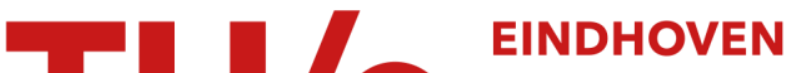

\section{Speed and efficiency of femtosecond spin current injection into a nonmagnetic material}

\section{Citation for published version (APA):}

Hofherr, M., Maldonado, P., Schmitt, O., Berritta, M., Bierbrauer, U., Sadashivaiah, S., Schellekens, A. J., Koopmans, B., Steil, D., Cinchetti, M., Stadtmüller, B., Oppeneer, P. M., Mathias, S., \& Aeschlimann, M. (2017). Speed and efficiency of femtosecond spin current injection into a nonmagnetic material. Physical Review $B$, 96(10), 1-6. [100403]. https://doi.org/10.1103/PhysRevB.96.100403

DOI:

10.1103/PhysRevB.96.100403

Document status and date:

Published: 14/09/2017

\section{Document Version:}

Publisher's PDF, also known as Version of Record (includes final page, issue and volume numbers)

\section{Please check the document version of this publication:}

- A submitted manuscript is the version of the article upon submission and before peer-review. There can be important differences between the submitted version and the official published version of record. People interested in the research are advised to contact the author for the final version of the publication, or visit the $\mathrm{DOI}$ to the publisher's website.

- The final author version and the galley proof are versions of the publication after peer review.

- The final published version features the final layout of the paper including the volume, issue and page numbers.

Link to publication

\section{General rights}

Copyright and moral rights for the publications made accessible in the public portal are retained by the authors and/or other copyright owners and it is a condition of accessing publications that users recognise and abide by the legal requirements associated with these rights.

- Users may download and print one copy of any publication from the public portal for the purpose of private study or research.

- You may not further distribute the material or use it for any profit-making activity or commercial gain

- You may freely distribute the URL identifying the publication in the public portal.

If the publication is distributed under the terms of Article 25fa of the Dutch Copyright Act, indicated by the "Taverne" license above, please follow below link for the End User Agreement:

www.tue.nl/taverne

Take down policy

If you believe that this document breaches copyright please contact us at:

openaccess@tue.nl

providing details and we will investigate your claim. 


\title{
Speed and efficiency of femtosecond spin current injection into a nonmagnetic material
}

\author{
M. Hofherr, ${ }^{1,2,{ }^{*}}$ P. Maldonado, ${ }^{3}$ O. Schmitt, ${ }^{1}$ M. Berritta, ${ }^{3}$ U. Bierbrauer, ${ }^{1}$ S. Sadashivaiah, ${ }^{1}$ A. J. Schellekens, ${ }^{4}$ B. Koopmans, ${ }^{4}$ \\ D. Steil,${ }^{5}$ M. Cinchetti, ${ }^{6}$ B. Stadtmüller,,${ }^{1,2}$ P. M. Oppeneer, ${ }^{3}$ S. Mathias, ${ }^{5,7, \dagger}$ and M. Aeschlimann ${ }^{1}$ \\ ${ }^{1}$ University of Kaiserslautern, Erwin-Schroedinger Strasse 46, D-67663 Kaiserslautern, Germany \\ ${ }^{2}$ Graduate School Materials Science in Mainz, Staudinger Weg 9, D-55128 Mainz, Germany \\ ${ }^{3}$ Department of Physics and Astronomy, Uppsala University, P.O. Box 516, S-75120 Uppsala, Sweden \\ ${ }^{4}$ Department of Applied Physics, Center for NanoMaterials (cNM), Eindhoven University of Technology, P.O. Box 513, \\ NL-5600 MB Eindhoven, The Netherlands \\ ${ }^{5}$ Georg-August-Universität Göttingen, I. Physikalisches Institut, Friedrich-Hund-Platz 1, D-37077 Göttingen, Germany \\ ${ }^{6}$ Experimentelle Physik VI, Technische Universität Dortmund, D-44221 Dortmund, Germany \\ ${ }^{7}$ International Center for Advanced Studies of Energy Conversion (ICASEC), Georg-August-Universität Göttingen, \\ D-37077 Göttingen, Germany
}

(Received 15 May 2017; published 14 September 2017)

\begin{abstract}
We investigate femtosecond spin injection from an optically excited Ni top layer into an Au bottom layer using time-resolved complex magneto-optical Kerr effect (C-MOKE) measurements. Employing the C-MOKE formalism, we are able to follow layer-resolved demagnetization in $\mathrm{Ni}$ and the simultaneous spin injection into the adjacent $\mathrm{Au}$ film, both occurring within $\sim 40 \mathrm{fs}$. We confirm the ballistic to diffusive propagation of the spin transfer process with $a b$ initio theory and superdiffusive transport calculations. In particular, our combined experimental-theoretical effort does allow us to quantify the so far elusive amount of spin injection, and therefore the spin injection efficiency at the interface.
\end{abstract}

DOI: 10.1103/PhysRevB.96.100403

Two decades after the pioneering work of Beaurepaire et al. [1], the fundamental processes of ultrafast laserinduced demagnetization are still heavily debated. Numerous experiments have been conducted, in addition to theoretical efforts, to elucidate the underlying microscopic mechanisms that can result in a surprisingly fast loss of magnetic order on a femtosecond (fs) time scale [2-6]. The microscopic mechanisms responsible for this photoinduced ultrafast loss of magnetic order can be classified as spin-flip scattering phenomena [2,7,8] and spin transport phenomena [9-12]. Today we know that both processes clearly contribute to the ultrafast demagnetization and that the contributions of both mechanisms depend on the details of the material system and multilayer structure [8,12-15]. In particular, optically induced spin transport via superdiffusive spin currents [4] yields a great potential for further applications. Instead of electrically generated spin currents in a ferromagnetic electrode via the spin Hall effect or other approaches, which are limited to time scales of today's electronic devices (ns), optically generated spin currents could provide the platform for spin current generation and spin current injection in nonmagnetic materials within a few hundreds of femtoseconds, thereby pushing spintronics to the ultrafast time scale.

However, the success of implementing optically generated spin currents into spintronic devices depends on our ability to predict the fundamental properties of the spin currents, such as generation efficiency, spin injection efficiency, and the lifetime of optically induced spin currents in nonmagnetic materials such as $\mathrm{Cu}$ or $\mathrm{Au}$. While spin currents have been proven to be relevant on femtosecond time scales $[11,15,16]$, only few details are known about the amount of optically induced spin currents or spin injection efficiency across various interfaces.

\footnotetext{
*barkowsm@physik.uni-kl.de

†smathias@uni-goettingen.de
}

This knowledge gap is mainly caused by the requirement to use fairly complex layer-specific ultrafast techniques to study such spin currents, and only a limited number of experimental approaches have so far been realized that fulfill this need. In particular, spin- and time-resolved two-photon photoemission [17] has been shown to be a powerful method to study metal-semiconductor interfaces, while fs light sources with energies in the extreme ultraviolet and soft $\mathrm{x}$-ray regime have been employed to gain layer specificity in metallic multilayer systems $[9,13,18-23]$. However, the latter techniques either require large-scale photon facilities or are experimentally fairly challenging.

In contrast, in this Rapid Communication, we apply the most commonly used technique to study ultrafast magnetization dynamics, i.e., the time-resolved magneto-optical Kerr effect (TR-MOKE) using visible light. For this technique, it was widely assumed that the measured signal contains only a weighted average response of the entire material. However, as it was shown by Hamrle et al. in 2002, one can overcome this limitation by taking advantage of the complex nature of the Kerr response, which can be represented by the complex Kerr angle $\Theta=\theta+i \varepsilon$ [24]. This so-called complex MOKE (C-MOKE) was very recently realized in a time-resolved experiment by Schellekens et al. [25]. Several experiments have been carried out since then which have shown both the proposed depth sensitivity [26] as well as the element sensitivity [25] of the C-MOKE technique. However, to disentangle the different contributions originating from different layers via the C-MOKE technique, so far one mostly has had to rely on a very specific sample geometry consisting of magnetic multilayer films with (at least) two separated ferromagnetic layers. Here, in contrast, we demonstrate the feasibility of C-MOKE for the study of a broader class of multilayer structures, and in our case a ferromagnetic-nonmagnetic bilayer system.

In this Rapid Communication, through the use of the layer-specific, femtosecond C-MOKE technique, we are able 


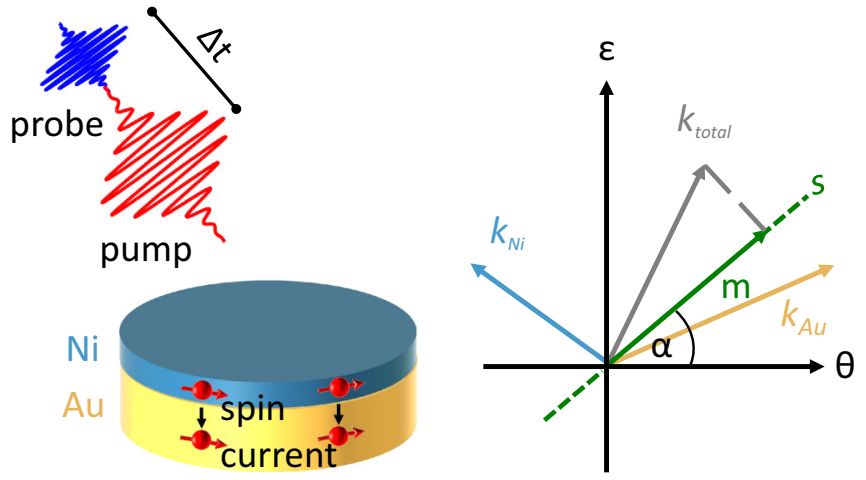

FIG. 1. Schematic overview of the C-MOKE measurement: The spin current excited by the pump pulse in the Ni film give rise to a MOKE signal of Au with a different contribution to the Kerr rotation and ellipticity as compared to $\mathrm{Ni}$. The C-MOKE measurement corresponds to a projection of the total Kerr vector of the sample system onto a projection axis $s$ in the complex plane.

to follow the injection of spin currents from a ferromagnetic into a nonmagnetic layer, which we find to occur on time scales as fast as $\sim 40 \mathrm{fs}$. Since the magnetic signal in the nonmagnetic Au layer only stems from injected spin carriers from $\mathrm{Ni}$, we can directly quantify the amount of spin current via a comparison with $a b$ initio calculations of the Au MOKE response. We find that the spin current injection efficiency at the interface is larger than $\sim 50 \%$.

The investigated sample is a metallic bilayer stack with $10 \mathrm{~nm} \mathrm{Ni}$ on top of a $150 \mathrm{~nm}$ Au film. In addition, Ni is capped with $10 \mathrm{~nm} \mathrm{SiO}_{2}$ to avoid oxidation of the ferromagnetic $\mathrm{Ni}$ film over time. To exclude any oxidation of the Ni layer during the capping procedure, we employed a nonreactive sputtering procedure in which the capping layer is directly deposited from a $\mathrm{SiO}_{2}$ sputtering target, i.e., without oxygen atmosphere. Similarly, all layers have been sputter deposited on an $\mathrm{MgO}$ substrate. In our time-resolved complex Kerr setup, we use a bichromatic pump-probe scheme (pump at $800 \mathrm{~nm}$, probe at $400 \mathrm{~nm}$ ) with a repetition rate of $1 \mathrm{kHz}$ and a full width at half maximum (FWHM) of the cross correlation of the pump and probe pulse of $\sim 75 \mathrm{fs}$ at the sample position. The experiment's principle is depicted in Fig. 1. The MOKE response of the material to the incoming linearly polarized light pulse is given by both a rotation $\theta$ of the polarization axis as well as a change of its ellipticity $\varepsilon$. These two real quantities are combined in a complex number, the so-called complex Kerr angle $\Theta=\theta+i \varepsilon$, which can be visualized as a vector $\boldsymbol{k}$ in a Cartesian coordinate system. A reduction of the magnetization is then represented by a reduction of the length of the Kerr vector $\boldsymbol{k}$. If there is more than one element in the sample system, such as $\mathrm{Ni}$ and $\mathrm{Au}$ in our case, the two individual Kerr vectors $\boldsymbol{k}_{\mathrm{Ni}}$ and $\boldsymbol{k}_{\mathrm{Au}}$ add as a vector sum to a total Kerr vector $\boldsymbol{k}_{\text {total }}=\boldsymbol{k}_{\mathrm{Ni}}+\boldsymbol{k}_{\mathrm{Au}}$. A MOKE measurement $m$ corresponds then to the absolute value of the orthogonal projection of this total Kerr vector onto a detection axis $\boldsymbol{s}: m=\boldsymbol{k}_{\text {total }} \cdot \boldsymbol{s}=\left|\boldsymbol{k}_{\text {total }}\right| \cos (\alpha)$. Using dedicated optics, we can freely choose an arbitrary detection axis in our experiments. This approach opens up the possibility to disentangle contributions from different elements/layers, if their corresponding Kerr vectors point in different directions.
For instance, one can choose the detection axis to be orthogonal to one of the Kerr vectors (i.e., elements or layers), so that the projection of this vector on the axis is zero and therefore this particular element/layer does not contribute to the measured data. We call this the "orthogonal data set." A more detailed derivation as well as information about the experimental realization can be found in Refs. [24,25].

For the interpretation of the collected C-MOKE data, it is necessary to distinguish between element dependence [25] and depth dependence [26,27] of the C-MOKE method. In our case, where we want to make use of the element dependence to gain layer specificity, we fabricated a sufficiently thin Ni layer, where the depth-dependent dynamics in $\mathrm{Ni}$ can be neglected [see Supplemental Material (SM) [28]]. Therefore, we can approximate that the magneto-optical response of a $10-\mathrm{nm} \mathrm{Ni}$ film can be described by a single complex Kerr vector pointing in a well-defined single direction in the complex plane. Consequently, in a Ni-orthogonal data set, where we are insensitive to $\mathrm{Ni}$, we are then able to extract the photoinduced transient magnetic signal that stems from Au. It is important to note that this only holds true if the $\mathrm{Ni}$ and Au Kerr vectors solely change their length but keep a fixed orientation in the complex plane upon ultrafast optical excitation. This, however, can be verified by a reference experiment that is shown in the SM [28].

The orientation of the complex Kerr angle of the undisturbed Ni film can be deduced by a static measurement of the absolute Kerr signal, i.e., the difference between positive and negative saturation signals, as a function of the complex Kerr angle. The largest Kerr signal will be obtained when the $\mathrm{Ni}$ Kerr vector is parallel to the detection axis. We call this detector configuration the "Ni-parallel" projection. The Ni-orthogonal data set, which does not contain any Ni signal, is correspondingly rotated by $90^{\circ}$. Figure 2 shows the Ni-parallel (left) and Ni-orthogonal (right) time-resolved C-MOKE traces of the optically induced magnetization dynamics. The Ni-parallel signal exhibits the shape of a typical transient demagnetization with a quenching level of $87 \pm 1 \%$. The Ni-orthogonal signal corresponds to the magnetization dynamics in Au and first increases after optical excitation, followed by a decay of the magnetic signal which even reverses its sign on longer time scales. We carry out exponential fits to the data with a single exponential decay in the case of $\mathrm{Ni}$ and an exponential increase followed by an exponential decay for the $\mathrm{Ni}$-orthogonal case that we assign to the spin dynamics in Au. Both fit functions were convolved with a Gaussian with a FWHM of $75 \mathrm{fs}$, representing the experimental time smear at the position of the sample. The demagnetization time for the Ni-parallel data set yields an extremely fast demagnetization constant of $42 \pm 8 \mathrm{fs}$. Although this signal has the maximum contribution from the Ni layer, it should be noted that it is not free of any Au signal, since the Au vector is not necessarily perpendicular to this detection axis. Nevertheless, since the Au signal is an order of magnitude smaller for all measured Kerr angles (see an overview of all measured Kerr signals in the SM [28]), the extracted time constant is representative of the Ni dynamics. The fit to the Ni-orthogonal data set, which in contrast shows the Au signal only, yields a rise time for the magnetization of $42 \pm 35 \mathrm{fs}$ and an exponential decay time of $255 \pm 70 \mathrm{fs}$. We note that the Ni demagnetization exponential time constant is within error identical to the Au rise exponential time constant. The straightforward interpretation of these data is that ultrafast optical excitation initiates the 

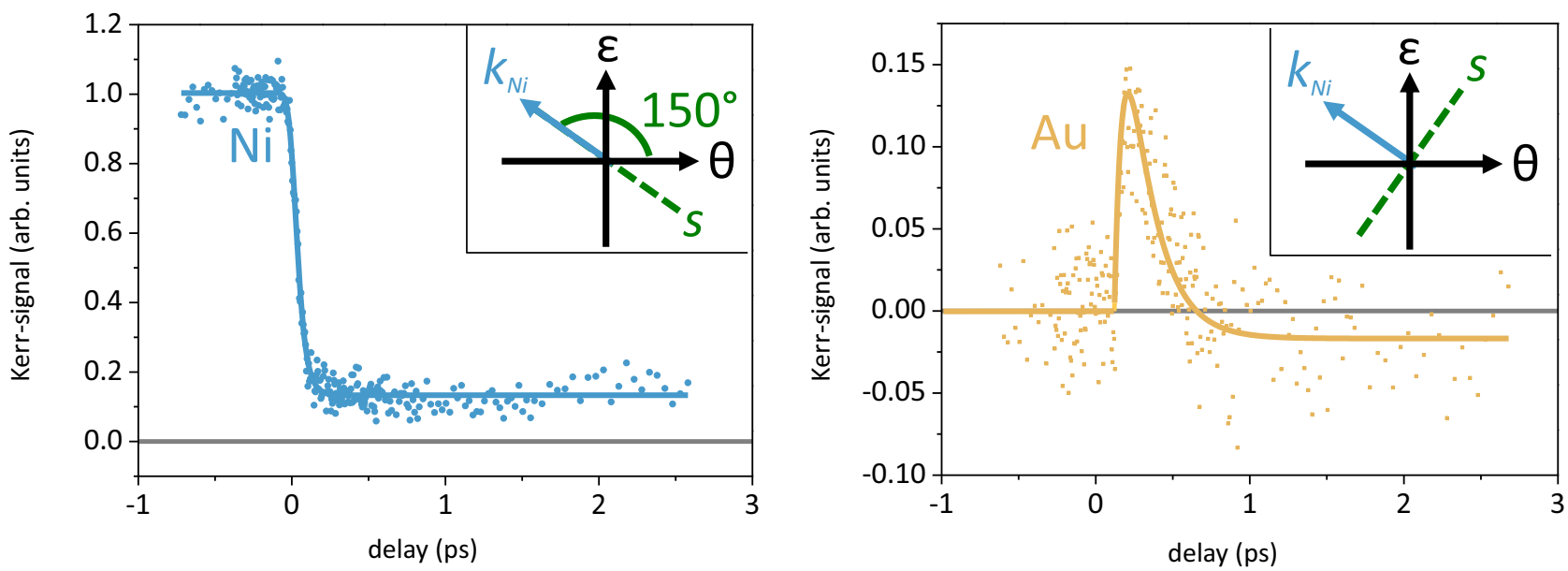

FIG. 2. Left: Normalized Kerr signal at the detection angle with the maximum signal before time zero corresponding to the highest Ni contribution. Right: Normalized Kerr signal at the detection angle with zero signal before time zero corresponding to the Au contribution. The solid lines are fits to the data with an exponential decay (Ni) and an exponential increase followed by an exponential decay (Au).

well-known superdiffusive spin currents from the Ni top layer which are instantaneously injected into the Au bottom layer, and that C-MOKE layer-specifically allows us to separate these dynamics. As already pointed out, however, a quantitative analysis of the amount of injected spins and a determination of the spin current injection efficiency has so far been elusive.

In our next step, we therefore aim to quantify the amount of injected spin carriers from the Ni to the Au layer. Here, our specific sample geometry is of advantage, since the magnetic Kerr signal from the initially nonmagnetic Au layer is completely induced by the spin current process, which allows a straightforward comparison with theory. For the quantification, we first need to know the magneto-optical constants of $\mathrm{Au}$ for the wavelength of the probe pulse used in our experiment $(400 \mathrm{~nm})$. We then can relate the Kerr signal to the injected magnetic moment. To this end we have performed $a b$ initio density-functional theory calculations of the magneto-optical response [29] of magnetized fcc Au (see SM [28]). This yields a rotation $\theta^{\mathrm{Au}}$ of $-0.0092^{\circ}$ and an ellipticity $\varepsilon^{\mathrm{Au}}$ of $-0.0055^{\circ}$ per $0.01 \mu_{\mathrm{B}}$ /atom. After calibrating the detector system with the Ni signal, we are able to determine the maximum injected moment in our experiment at a pump-probe delay of 120 fs (peak position in the right graph of Fig. 2), which is $0.015 \pm 0.002 \mu_{\mathrm{B}} /$ atom.

At first sight, the amount of spin carriers in $\mathrm{Au}$ is much lower than one would have expected in the case where all spin carriers lost in $\mathrm{Ni}$ upon demagnetization would be transferred to $\mathrm{Au}\left(87 \%\right.$ of $0.6 \mu_{\mathrm{B}}$ /atom, i.e., $0.52 \mu_{\mathrm{B}}$ /atom). To understand the seemingly small induced magnetization in $\mathrm{Au}$, we note that there exist several effects that lower the measured time-dependent injected moment into $\mathrm{Au}$, which we schematically depict in the inset of Fig. 3. First, the penetration depth of the 400-nm probe light into the Au layer is on the order of $20 \mathrm{~nm}$. Hence, we probe only a small part of the 150-nm-thick Au film, and therefore not the total amount of the injected spin carriers into the Au film at all times. Second, and even more important, is the high mobility of the spin carriers in Au. Therefore, the spins injected within the first tens of femtoseconds have at 120 fs already left the probed area and propagated deeper into the thick Au film. Third, the injected spins have a finite lifetime in the Au layer, and the spin polarization of the Au film induced by the injected spin carriers will decay over time due to spin-flip scattering processes. This effect also lowers the transient C-MOKE signal of Au.

To confirm our model, we performed a simulation of this sample system using the superdiffusive transport model $[4,30]$ with an additional nonzero spin-flip scattering probability

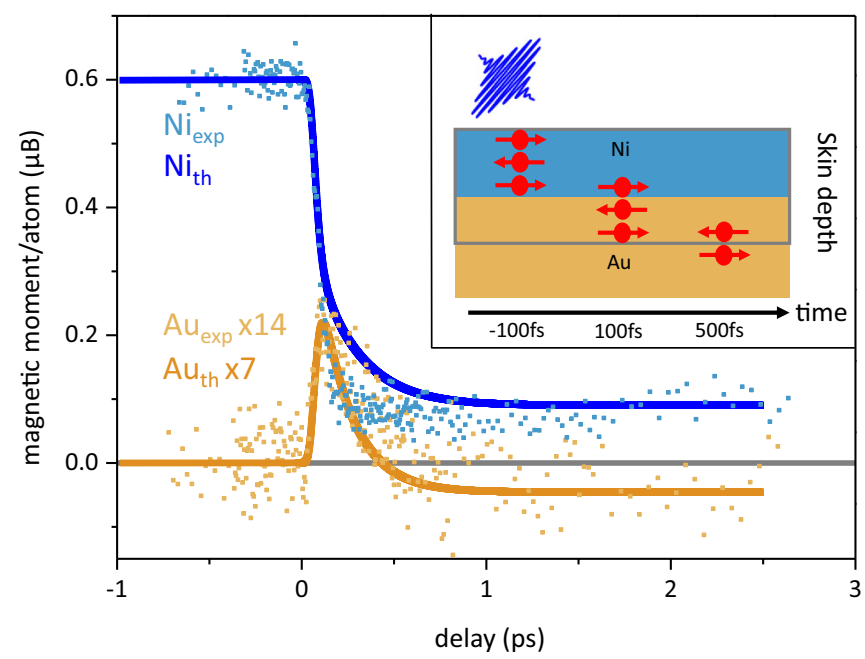

FIG. 3. Comparison between a superdiffusive spin-transport model including a spin relaxation in $\mathrm{Au}$ (solid line) and the experimental data (dots). The experimental Au data were multiplied by a factor of 14, and the theoretical Au data by a factor of 7 for better visibility. Inset: Schematic illustration of the measured Au signal. Before the pump arrives there are no spins in the $\mathrm{Au}$ ( $-100 \mathrm{fs}$ ). After the optical excitation, the spins start to propagate into the Au. In this process, about $50 \%$ of the polarization is lost due to spin-flip scattering at the interface. The residual polarization gives rise to a MOKE signal in Au (100 fs). Subsequently, the spin carriers leave the probed region that is limited by the skin depth of the blue probe light and the signal decreases. In addition, the spin polarization in $\mathrm{Au}$ is reduced via spin relaxation in the Au film (500 fs), which finally leads to an excess of minority spins and a magnetization reversal. 
in Au. The spin injection into Au occurs only due to the demagnetization in Ni (see SM [28]). Therefore, the injected spins in the Au film are solely given via the demagnetization of the Ni film, and the spin relaxation in Au is modeled by the spin flow of spin carriers out of the probed area as well as by spinflip processes. Including both spin relaxation channels in $\mathrm{Au}$, we can fully describe the experimentally observed decay of the $\mathrm{C}-\mathrm{MOKE}$ signal in Au. This is illustrated in Fig. 3 in which the theoretically modeled transient magnetization traces of Au and $\mathrm{Ni}$ are superimposed onto the experimental C-MOKE traces of both layers. The experimental and theoretical data show a good quantitative agreement concerning the exponential decay and rise-time constants of the magnetic signal in $\mathrm{Ni}$ and $\mathrm{Au}$. Minor differences between the experimental and simulated demagnetization traces of Ni between 50 and 500 fs can be attributed to an additional reduction of magnetization in $\mathrm{Ni}$ by spin-flip scattering processes which are not considered in our calculation. The amount of lost spins in $\mathrm{Ni}$ and injected spins in Au can be quantified by comparing the overall amplitude of the experimental and simulated demagnetization traces for both materials. We find a loss of magnetic moments in $\mathrm{Ni}$ of $0.52 \pm 0.02 \mu_{\mathrm{B}} /$ atom, but only an increase of magnetic moments of $0.25 \pm 0.07 \mu_{\mathrm{B}}$ /atom in Au. Hence, the amount of spin moments that is lost in $\mathrm{Ni}$, but not transferred to the $\mathrm{Au}$ substrate, is $0.27 \pm 0.09 \mu_{\mathrm{B}}$ /atom. The latter contributions are therefore spins that are lost either in $\mathrm{Ni}$ itself during the demagnetization process via, e.g., Elliott-Yafet spin-flip scattering, or spins that are initially part of the optically excited spin current in $\mathrm{Ni}$ but which are scattered directly at the $\mathrm{Ni} / \mathrm{Au}$ interface [31].

The amount of possible spin-flip scattering inside the $\mathrm{Ni}$ film, i.e., the former contribution to the loss of magnetic moment, can be deduced via a reference experiment. For this purpose, we now compare the measured demagnetization time constants for $\mathrm{Ni}$ on $\mathrm{Au}$, where spin current injection into the $\mathrm{Au}$ substrate is allowed, and $\mathrm{Ni}$ on $\mathrm{MgO}$, where the demagnetization can only be caused by spin-flip scattering within the Ni film, and not by spin transport. Carrying out these two comparative measurements, we find that $\mathrm{Ni}$ on $\mathrm{MgO}$ demagnetizes with a time constant of $120 \pm 14 \mathrm{fs}$ (see SM [28] and compare also with Refs. [2,7]), which is almost three times larger than in the case of $\mathrm{Ni}$ on Au with $42 \pm 8 \mathrm{fs}$. This significant difference in the demagnetization times of $\mathrm{Ni}$ on different substrates clearly shows that the loss of magnetic moment via spin-flip scattering takes considerably longer than the dominant demagnetization mechanism for $\mathrm{Ni}$ on $\mathrm{Au}$. As ultrafast spin transport is the main difference between both bilayer systems, we have substantial evidence that the demagnetization dynamics in the case of $\mathrm{Ni}$ on $\mathrm{Au}$ is dominantly driven by spin currents. This conclusion is also in line with our previous explanation that minor discrepancies between the experimental and simulated demagnetization traces in Fig. 3 can be attributed to spin-flip scattering processes occurring on time scales larger than 50 fs.

Based on the finding that the demagnetization in $\mathrm{Ni}$ is almost exclusively caused by spin currents from $\mathrm{Ni}$ into $\mathrm{Au}$, we conclude that spin-flip scattering processes at the interface and in the $\mathrm{Ni}$ film are responsible for the remaining 50\% loss of magnetic moment in the spin transfer process to the $\mathrm{Au}$ film. With a $\sim 50 \%$ loss of spin moment that we do not detect in Au, maximally every fourth electron must have flipped its spin in the transfer process. This corresponds to a spin current injection efficiency at the $\mathrm{Ni}-\mathrm{Au}$ interface of $50 \%$.

More precisely, this value is a quantification of the lower limit of the spin current injection efficiency from $\mathrm{Ni}$ into Au. It is calculated with the assumption that the loss of magnetic moments in $\mathrm{Ni}$ on $\mathrm{Au}$ is solely driven by optically generated spin currents which all move towards the $\mathrm{Ni}-\mathrm{Au}$ interface. Although this is a good approximation (as deduced by the demagnetization time constants of $\mathrm{Ni}$ on $\mathrm{Au}$ and $\mathrm{Ni}$ on $\mathrm{MgO}$ ), it is not perfect. In real systems, additional microscopic mechanisms such as Elliott-Yafet spin-flip scattering [32] and ultrafast magnon generation [33-36] can have additional contributions to the loss of magnetic moments in $3 d$ ferromagnetic materials. These effects reduce the amount of magnetic moments transported towards the $\mathrm{Ni}-\mathrm{Au}$ interface and hence result in a larger spin injection efficiency into Au. However, as a precise quantification of these contributions to the overall loss of magnetic moments in $\mathrm{Ni}$ is rather challenging, we refrain from further speculations and conclude that the spin current injection efficiency from $\mathrm{Ni}$ into $\mathrm{Au}$ is larger than $50 \%$.

Finally, we want to focus on the dynamics on time scales beyond 500 fs. Here, the experimental and theoretical data show a very distinct behavior for the measured Kerr signal in $\mathrm{Au}$, i.e., a reversal of the magnetization. This is remarkable since in the modeling we use $a b$ initio calculated spin lifetimes and velocities in Au that are identical for majority- and minority-spins [37]. Initially, many majority-spin electrons having a high energy that are injected into the Au layer have a high probability of undergoing a spin-flip scattering process. Minority-spin electrons are injected as well, but, since these have a shorter lifetime in $\mathrm{Ni}$, they have already lost energy and are delayed. Their later arrival, together with the high rate of spin-flip scattering of majority-spin electrons, leads to an excess of minority-spins in the field of view and hence to a negative transient magnetization, as can be seen from Fig. 3. Our interpretation of these phenomena is fully supported by previous investigations of optically induced spin currents in Au films [11].

In conclusion, we find that we are able to use C-MOKE to follow layer-resolved femtosecond injection of superdiffusive spin currents from a Ni film into a nonmagnetic Au substrate. A direct comparison of the demagnetization time constants of $\mathrm{Ni}$ on different substrates provides substantial evidence that the demagnetization dynamics of $\mathrm{Ni} / \mathrm{Au}$ is mainly caused by optically generated spin currents. Via comparison with theory, we are able to quantify the amount of injected moment from the $\mathrm{Ni}$ to the $\mathrm{Au}$ to be $0.25 \mu_{\mathrm{B}} /$ atom, which corresponds to $\sim 50 \%$ of the lost moment in $\mathrm{Ni}$ and to a spin current injection efficiency into Au of 50\%. Taking into account further demagnetization mechanisms such as Elliott-Yafet spin-flip scatterings and ultrafast magnon generation, we are able to conclude that this value is a lower limit of the spin current injection efficiency at the $\mathrm{Ni}-\mathrm{Au}$ polycrystalline interface, and that the real injection efficiency must be even larger than $50 \%$.

Moreover, our combined experimental and theoretical approach provides the ideal methodology for future investigations of fs-spin current injection in spintronic assembles with the goal to engineer interfaces for the highest spin current injection efficiency. Highly interesting approaches to 
enhance the spin current injection efficiency across interfaces are either to optimize the morphology of the interface by employing different growth conditions or to choose materials with matching Fermi surfaces. Such investigations will be essential for the successful implementation of ultrafast spin currents in spintronic applications.

S.M. and D.S. acknowledge support from the SFB 1073 "Atomic scale control of energy conversion". M.A., B.S., and M.H. acknowledge support from the SFB/TRR 173 "Spin+X"
(Project B03). M.H. acknowledges support as a recipient of a DFG-fellowship/DFG-funded position through the Excellence Initiative by the Graduate School of Excellence MAINZ (Excellence Initiative No. DFG/GSC 266). B.S. thankfully acknowledges financial support from the Graduate School of Excellence MAINZ (Excellence Initiative No. DFG/GSC 266). M.B., P.M., and P.M.O. acknowledge support from the Swedish Research Council (VR), the K. and A. Wallenberg Foundation (Grant No. 2015.0060), and the Swedish National Infrastructure for Computing (SNIC).
[1] E. Beaurepaire, J.-C. Merle, A. Daunois, and J.-Y. Bigot, Phys. Rev. Lett. 76, 4250 (1996).

[2] B. Koopmans, G. Malinowski, F. Dalla Longa, D. Steiauf, M. Fähnle, T. Roth, M. Cinchetti, and M. Aeschlimann, Nat. Mater. 9, 259 (2010).

[3] M. Krauß, T. Roth, S. Alebrand, D. Steil, M. Cinchetti, M. Aeschlimann, and H. C. Schneider, Phys. Rev. B 80, 180407 (2009).

[4] M. Battiato, K. Carva, and P. M. Oppeneer, Phys. Rev. Lett. 105, 027203 (2010).

[5] J.-Y. Bigot, M. Vomir, and E. Beaurepaire, Nat. Phys. 5, 515 (2009).

[6] W. Hübner and G. Zhang, J. Magn. Magn. Mater. 189, 101 (1998).

[7] C. La-O-Vorakiat, E. Turgut, C. A. Teale, H. C. Kapteyn, M. M. Murnane, S. Mathias, M. Aeschlimann, C. M. Schneider, J. M. Shaw, H. T. Nembach, and T. J. Silva, Phys. Rev. X 2, 011005 (2012).

[8] A. J. Schellekens, W. Verhoeven, T. N. Vader, and B. Koopmans, Appl. Phys. Lett. 102, 252408 (2013).

[9] D. Rudolf, C. La-O-Vorakiat, M. Battiato, R. Adam, J. M. Shaw, E. Turgut, P. Maldonado, S. Mathias, P. Grychtol, H. T. Nembach, T. J. Silva, M. Aeschlimann, H. C. Kapteyn, M. M. Murnane, C. M. Schneider, and P. M. Oppeneer, Nat. Commun. 3, 1037 (2012).

[10] G. Malinowski, F. Dalla Longa, J. H. H. Rietjens, P. V. Paluskar, R. Huijink, H. J. M. Swagten, and B. Koopmans, Nat. Phys. 4, 855 (2008).

[11] A. Melnikov, I. Razdolski, T. O. Wehling, E. T. Papaioannou, V. Roddatis, P. Fumagalli, O. Aktsipetrov, A. I. Lichtenstein, and U. Bovensiepen, Phys. Rev. Lett. 107, 076601 (2011).

[12] A. Alekhin, I. Razdolski, N. Ilin, J. P. Meyburg, D. Diesing, V. Roddatis, I. Rungger, M. Stamenova, S. Sanvito, U. Bovensiepen, and A. Melnikov, Phys. Rev. Lett. 119, 017202 (2017).

[13] S. Mathias, C. La-O-Vorakiat, J. M. Shaw, E. Turgut, P. Grychtol, R. Adam, D. Rudolf, H. T. Nembach, T. J. Silva, M. Aeschlimann, C. M. Schneider, H. C. Kapteyn, and M. M. Murnane, J. Electron Spectrosc. Relat. Phenom. 189, 164 (2013).

[14] A. Eschenlohr, M. Battiato, P. Maldonado, N. Pontius, T. Kachel, K. Holldack, R. Mitzner, A. Föhlisch, P. M. Oppeneer, and C. Stamm, Nat. Mater. 12, 332 (2013).

[15] E. Turgut, C. La-O-Vorakiat, J. M. Shaw, P. Grychtol, H. T. Nembach, D. Rudolf, R. Adam, M. Aeschlimann, C. M. Schneider, T. J. Silva, M. M. Murnane, H. C. Kapteyn, and S. Mathias, Phys. Rev. Lett. 110, 197201 (2013).
[16] A. J. Schellekens, K. C. Kuiper, R .R. J. C. de Wit, and B. Koopmans, Nat. Commun. 5, 4333 (2014).

[17] M. Cinchetti, K. Heimer, J.-P. Wüstenberg, O. Andreyev, M. Bauer, S. Lach, C. Ziegler, Y. Gao, and M. Aeschlimann, Nat. Mater. 8, 115 (2009)

[18] C. Stamm, T. Kachel, N. Pontius, R. Mitzner, T. Quast, K. Holldack, S. Khan, C. Lupulescu, E. F. Aziz, M. Wietstruk, H. A. Dürr, and W. Eberhardt, Nat. Mater. 6, 740 (2007).

[19] I. Radu, K. Vahaplar, C. Stamm, T. Kachel, N. Pontius, H. A. Dürr, T. A. Ostler, J. Barker, R. F. L. Evans, R. W. Chantrell, A. Tsukamoto, A. Itoh, A. Kirilyuk, T. Rasing, and A. V. Kimel, Nature (London) 472, 205 (2011).

[20] C. La-O-Vorakiat, M. Siemens, M. M. Murnane, H. C. Kapteyn, S. Mathias, M. Aeschlimann, P. Grychtol, R. Adam, C. M. Schneider, J. M. Shaw, H. Nembach, and T. J. Silva, Phys. Rev. Lett. 103, 257402 (2009).

[21] S. Mathias, C. La-O-Vorakiat, P. Grychtol, P. Granitzka, E. Turgut, J. M. Shaw, R. Adam, H. T. Nembach, M. E. Siemens, S. Eich, C. M. Schneider, T. J. Silva, M. Aeschlimann, M. M. Murnane, and H. C. Kapteyn, Proc. Natl. Acad. Sci. U.S.A. 109, 4792 (2012).

[22] B. Pfau, S. Schaffert, L. Müller, C. Gutt, A. Al-Shemmary, F. Büttner, R. Delaunay, S. Dusterer, S. Flewett, R. Frömter, J. Geilhufe, E. Guehrs, C. M. Günther, R. Hawaldar, M. Hille, N. Jaouen, A. Kobs, K. Li, J. Mohanty, H. Redlin, W. F. Schlotter, D. Stickler, R. Treusch, B. Vodungbo, M. Kläui, H. P. Oepen, J. Lüning, G. Grübel, and S. Eisebitt, Nat. Commun. 3, 1100 (2012).

[23] F. Willems, C. von Korff Schmising, D. Weder, C. M. Günther, M. Schneider, B. Pfau, S. Meise, E. Guehrs, J. Geilhufe, A. E. D. Merhe, E. Jal, B. Vodungbo, J. Lüning, B. Mahieu, F. Capotondi, E. Pedersoli, D. Gauthier, M. Manfredda, and S. Eisebitt, Struct. Dyn. 4, 14301 (2017).

[24] J. Hamrle, J. Ferré, M. Nývlt, and Š. Višňovský, Phys. Rev. B 66, 224423 (2002).

[25] A. J. Schellekens, N. de Vries, J. Lucassen, and B. Koopmans, Phys. Rev. B 90, 104429 (2014).

[26] J. Wieczorek, A. Eschenlohr, B. Weidtmann, M. Rösner, N. Bergeard, A. Tarasevitch, T. O. Wehling, and U. Bovensiepen, Phys. Rev. B 92, 174410 (2015).

[27] G. Traeger, L. Wenzel, and A. Hubert, Phys. Status Solidi A 131, 201 (1992).

[28] See Supplemental Material at http://link.aps.org/supplemental/ 10.1103/PhysRevB.96.100403 for a comparison of the demagnetization dynamics of $\mathrm{Ni}$ on the insulating substrate $\mathrm{MgO}$, for more details on the theoretical model, for a complete overview of the diversity in the Kerr signals at different complex angles, 
and for a detailed discussion on the depth and time dependency of the Kerr signal, which includes Refs. [4,26,29,37-42].

[29] P. M. Oppeneer, in Handbook of Magnetic Materials, edited by K. H. J. Buschow (Elsevier, Amsterdam, 2001), Vol. 13, pp. 229-422.

[30] M. Battiato, K. Carva, and P. M. Oppeneer, Phys. Rev. B 86, 024404 (2012).

[31] M. D. Stiles, J. Appl. Phys. 79, 5805 (1996).

[32] K. Carva, M. Battiato, D. Legut, and P. M. Oppeneer, Phys. Rev. B 87, 184425 (2013).

[33] E. Turgut, D. Zusin, D. Legut, K. Carva, R. Knut, J. M. Shaw, C. Chen, Z. Tao, H. T. Nembach, T. J. Silva, S. Mathias, M. Aeschlimann, P. M. Oppeneer, H. C. Kapteyn, M. M. Murnane, and P. Grychtol, Phys. Rev. B 94, 220408(R) (2016).

[34] S. Eich, M. Plötzing, M. Rollinger, S. Emmerich, R. Adam, C. Chen, H. C. Kapteyn, M. M. Murnane, L. Plucinski, D. Steil,
B. Stadtmüller, M. Cinchetti, M. Aeschlimann, C. M. Schneider, and S. Mathias, Sci. Adv. 3, 1602094 (2017).

[35] E. Carpene, H. Hedayat, F. Boschini, and C. Dallera, Phys. Rev. B 91, 174414 (2015).

[36] M. Haag, C. Illg, and M. Fähnle, Phys. Rev. B 90, 014417 (2014).

[37] V. P. Zhukov and E. V. Chulkov, Phys.-Usp. 52, 105 (2009).

[38] B. Koopmans, M. van Kampen, J. T. Kohlhepp, and W. J. M. de Jonge, Phys. Rev. Lett. 85, 844 (2000).

[39] P. M. Oppeneer, T. Maurer, J. Sticht, and J. Kübler, Phys. Rev. B 45, 10924 (1992).

[40] R. Mondal, M. Berritta, K. Carva, and P. M. Oppeneer, Phys. Rev. B 91, 174415 (2015).

[41] C.-Y. You and S.-C. Shin, Appl. Phys. Lett. 69, 1315 (1996).

[42] V. P. Zhukov, E. V. Chulkov, and P. M. Echenique, Phys. Rev. B 73, 125105 (2006). 